\title{
Genome-Wide Characterization and Analysis of bHLH Transcription Factors Related to Crocin Biosynthesis in Gardenia jasminoides Ellis (Rubiaceae)
}

\author{
Ya Tian $\mathbb{D}^{1,2}$ Xiangdong Pu $\mathbb{D}^{2},{ }^{2}$ Haoying Yu, ${ }^{2}$ Aijia Ji, ${ }^{3}$ Ranran Gao $\mathbb{D}^{2},{ }^{2}$ Yating $\mathrm{Hu},{ }^{2}$ \\ Zhichao Xu ${ }^{2}{ }^{2,4}$ and Hualei Wang ${ }^{1}$ \\ ${ }^{1}$ Key Laboratory of Propagation and Cultivation on Medicinal Plants, College of Agriculture, Guizhou University, \\ Guizhou 550025, China \\ ${ }^{2}$ Institute of Medicinal Plant Development, Chinese Academy of Medical Science, Peking Union Medical College, \\ Beijing 100193, China \\ ${ }^{3}$ School of Pharmaceutical Sciences, Guangzhou University of Chinese Medicine, Guangzhou 510006, China \\ ${ }^{4}$ Engineering Research Center of Chinese Medicine Resource, Ministry of Education, Beijing 100193, China
}

Correspondence should be addressed to Zhichao Xu; zcxu@implad.ac.cn and Hualei Wang; gzuwhl@126.com

Received 18 November 2019; Revised 29 February 2020; Accepted 12 March 2020; Published 6 April 2020

Academic Editor: Fengjie Sun

Copyright $\odot 2020$ Ya Tian et al. This is an open access article distributed under the Creative Commons Attribution License, which permits unrestricted use, distribution, and reproduction in any medium, provided the original work is properly cited.

\begin{abstract}
Crocins, enriched in Gardenia jasminoides fruits, have a pharmacological activity against central nervous system diseases, cardiovascular diseases, and cancer cell growth. The biosynthesis of crocins has been widely explored, but its regulatory mechanism remains unknown. Here, the basic helix-loop-helix (bHLH) transcription factors related to crocin biosynthesis were systematically identified on the basis of the genome of G. jasminoides. A total of 95 GjbHLH transcription factor genes were identified, and their phylogenetic analysis indicated that they could be classified into 23 subfamilies. The combination of genespecific $b H L H$ expression patterns, the coexpression analysis of biosynthesis genes, and the analysis of promoter sequences in crocin biosynthesis pathways suggested that nine bHLHs in G. jasminoides might negatively regulate crocin biosynthesis. This study laid a foundation for understanding the regulatory mechanism of crocin biosynthesis and the improvement and breeding of G. jasminoides varieties.
\end{abstract}

\section{Introduction}

Crocins, glucosyl esters of crocetin, belong to apocarotenoids, which are highly accumulated in mature fruits of Gardenia jasminoides [1] and stigma of Crocus sativus. Crocins have a curative effect on various types of central nervous system diseases, such as neurodegenerative diseases [2], and various types of cardiovascular system disease, such as hypertension [3]. Crocins also have pharmacological activities, including anticancer cell growth [4], anti-inflammation and antioxidation [5], antiplatelet aggregation [6], and antiobesity [7]. G. jasminoides, named Zhi Zi in traditional Chinese medicine and recorded in the Chinese pharmacopoeia, is commonly used for antioxidant activity, anti-inflammation, and detoxification [8]. G. jasminoides, belonging to the coffee family, is a famous ornamental plant, widely distributed around the world [9].

In G. jasminoides fruits, the biosynthesis of crocins begins with the cleavage of carotenoids under the activity of carotenoid cleavage dioxygenase (CCD) to produce crocetin dialdehyde. Then, aldehyde dehydrogenase (ALDH) catalyzes the transformation of crocetin dialdehyde to crocetin, which is the key precursor of crocins. Lastly, the glycosylation of different crocetins under the catalysis of UDPglucosyltransferase (UGT) produces various crocins (crocins I-V) (Figure S1). The genome of G. jasminoides has been finished, and the complete pathway of crocin biosynthesis including GjCCD4a, GjALDH2C3, GjUGT94E13, and GjUGT74F8 has been systematically elucidated (NCBI: VZDL00000000). However, the regulation of crocins 
produced in G. jasminoides remains unclear. The regulation of transcription factors (TFs) plays an important role in the biosynthesis of active compounds [10-14].

The bHLH superfamily, one of the largest TF families in plants, has been indicated to be related to plant development and stress response. The genome-wide identification and analysis of bHLH TFs have been reported in many plants, such as Arabidopsis thaliana [15], Oryza sativa [16], Solanum lycopersicum [17], Brassica rapa [18], Salvia miltiorrhiza [19], Panax ginseng [20], Vitis vinifera [21], Malus domestica [22], and Arachis hypogaea [23]. bHLH TFs can bind to E-box ( $5^{\prime}$-CANNTG- $\left.3^{\prime}\right)$, MYCATRD22 ( $5^{\prime}$-CACATG-3'), T/G-box ( $5^{\prime}$-AACGTG-3'), or G-box $\left(5^{\prime}\right.$-CACGTG- $\left.3^{\prime}\right)$ elements to regulate gene expression [24-26]. Based on transcriptome analysis of C. sativus, many transcription factors including two bHLH members were selected to be involved in the crocin biosynthesis and accumulation [27]. Furthermore, the functions of many bHLH proteins in plants have been indicated to regulate the biosynthesis of secondary metabolites. For example, in Panax notoginseng, PnbHLH1 improves triterpenoid biosynthesis by interacting with E-box core sequences in the promoter region of target genes [28]. In strawberry fruits, FvbHLH9 positively regulates anthocyanin biosynthesis by forming HY5-bHLH9 transcription complexes [29]. MYCs are important bHLH family members, and MYC2s from different species positively or negatively regulate the biosynthesis of active compounds. In A. thaliana, MYC2 negatively regulates indole glucosinolate biosynthesis during jasmonic acid (JA) signaling. Furthermore, MYC2 positively regulates ascorbate redox cycling and flavonoid biosynthesis to enhance tolerance to insect pests and oxidative stress [25]. In Taxus chinensis, three JAinducible MYC TFs, TcJAMYC1, TcJAMYC2, and TcJAMYC4, negatively regulate the paclitaxel biosynthesis [26].

The regulation of bHLH TFs in crocin biosynthesis has not been described in G. jasminoides. Here, we systematically selected bHLH TFs and identified the candidate bHLHs related to the regulation of crocin production based on the genome of G. jasminoides. Our results provided a basis for further studying the regulatory mechanism of crocin biosynthesis and the breeding of G. jasminoides varieties.

\section{Materials and Methods}

2.1. Plant Materials. The variety of G. jasminoides, named ZZ1-9, was selected and cultivated in the Chongqing Institute of Medicinal Implantation. The root, stem, leaf, young fruit, green fruit, and red fruit of G. jasminoides (ZZ1-9) were treated with liquid nitrogen and stored at $-80^{\circ} \mathrm{C}$ for subsequent experiments.

2.2. Identification of bHLH Genes and Sequence Feature Analysis. The bHLH sequences of Arabidopsis were downloaded from the Arabidopsis database (https://www .arabidopsis.org/), Ensembl Plants (http://plants.ensembl.org/ index.html), and NCBI (https://www.ncbi.nlm.nih.gov/), and the accession numbers of $A t b H L H$ genes are listed in Table S1. All GjbHLH genes were identified using the HMMER analysis of the bHLH domain (HLH: PF00010.26 or
bHLH-MYC_N: PF14215.6) against the G. jasminoides genome. The GjbHLH genes were manually corrected using a protein BLAST algorithm (http://blast.ncbi.nlm.nih.gov/Blast .cgi). The theoretical isoelectric point $(\mathrm{p} I)$ and the molecular weight $(\mathrm{Mw})$ of the GjbHLH proteins were predicted using the computed $\mathrm{pI} / \mathrm{Mw}$ tool on the ExPASy server (http://web .expasy.org/compute_pi/).

\subsection{Phylogenetic, Gene Structure, and MEME Motif Analyses.} A total of $638 \mathrm{bHLH}$ genes from Arabidopsis, poplar, rice, moss, and algae were classified into 32 subfamilies [30]. The protein sequences of the bHLH family from G. jasminoides and Arabidopsis were aligned using MUSCLE methods [31]. Then, the multiple sequence alignments were used to construct a Maximum Likelihood (ML) tree by MEGA 6.0 with the Jones-Taylor-Thornton model and 1000 bootstrap replicates [32]. The phylogenetic tree of the MYC subfamily and the bHLH3 subfamily was constructed using the same method, and bHLH15 subfamily members, GjbHLH15.1 and GjbHLH15.7, were chosen as the outgroup. The online Gene Structure Display Server (GSDS 2.0) (http:// gsds.cbi.pku.edu.cn) was used to investigate the gene structure based on each coding sequence (CDS) and the corresponding genomic sequence. The conserved motifs in bHLH TFs of G. jasminoides were identified using MEME (suite version 5.0.3) with the following criteria: 22 motifs, any number of repetitions of a motif, and an optimum width of 10-200 amino acids $[19,33]$.

2.4. Gene Expression Analysis. The RNA-Seq from six organs (root, stem, leaf, young fruit, green fruit, and red fruit) of $G$. jasminoides was performed. The RNA-Seq reads were mapped to the G. jasminoides genome using HISAT2 [34], and the expression levels of $b H L H$ genes were estimated on the basis of fragments per kilobase per million (FPKM) values using Cufflinks [35].

Total RNA was extracted from the root, stem, leaves, green fruits, and red fruits using an RNAprep Pure Plant Kit (TIANGEN Biotech, China) according to the manufacturer's instructions. The expression levels of GjbHLH candidate genes identified as putative regulators of crocin biosynthesis were confirmed by quantitative real-time reverse transcription PCR (qPCR) analysis in triplicate. Total RNA was reverse transcribed using a PrimeScript ${ }^{\mathrm{TM}}$ II 1 st Strand cDNA Synthesis Kit (TaKaRa, China). qPCR was performed with a TB Green ${ }^{\mathrm{TM}}$ premix Ex $\mathrm{Taq}^{\mathrm{TM}}$ (Tli RNaseH Plus) (TaKaRa, China) and conducted in triplicate using an Applied Biosystems 7500 Real-Time PCR system (Life Technologies, USA). The primers were designed using Primer Premier 6 (Table S2), with an amplicon size ranging from $150 \mathrm{bp}$ to $250 \mathrm{bp}$ and an optimal Tm of $55 \pm 5^{\circ} \mathrm{C}$. The actin gene was used as an internal reference, and red fruit was examined for comparison; $\mathrm{Ct}$ values were calculated to analyze the relative expression levels by using the $2^{-\Delta \Delta \mathrm{Ct}}$ method [36]. One-way ANOVA was performed with GraphPad Prism to detect the differences in candidate gene expression. ${ }^{*} P<0.05$ was considered to indicate statistical difference in expression. 


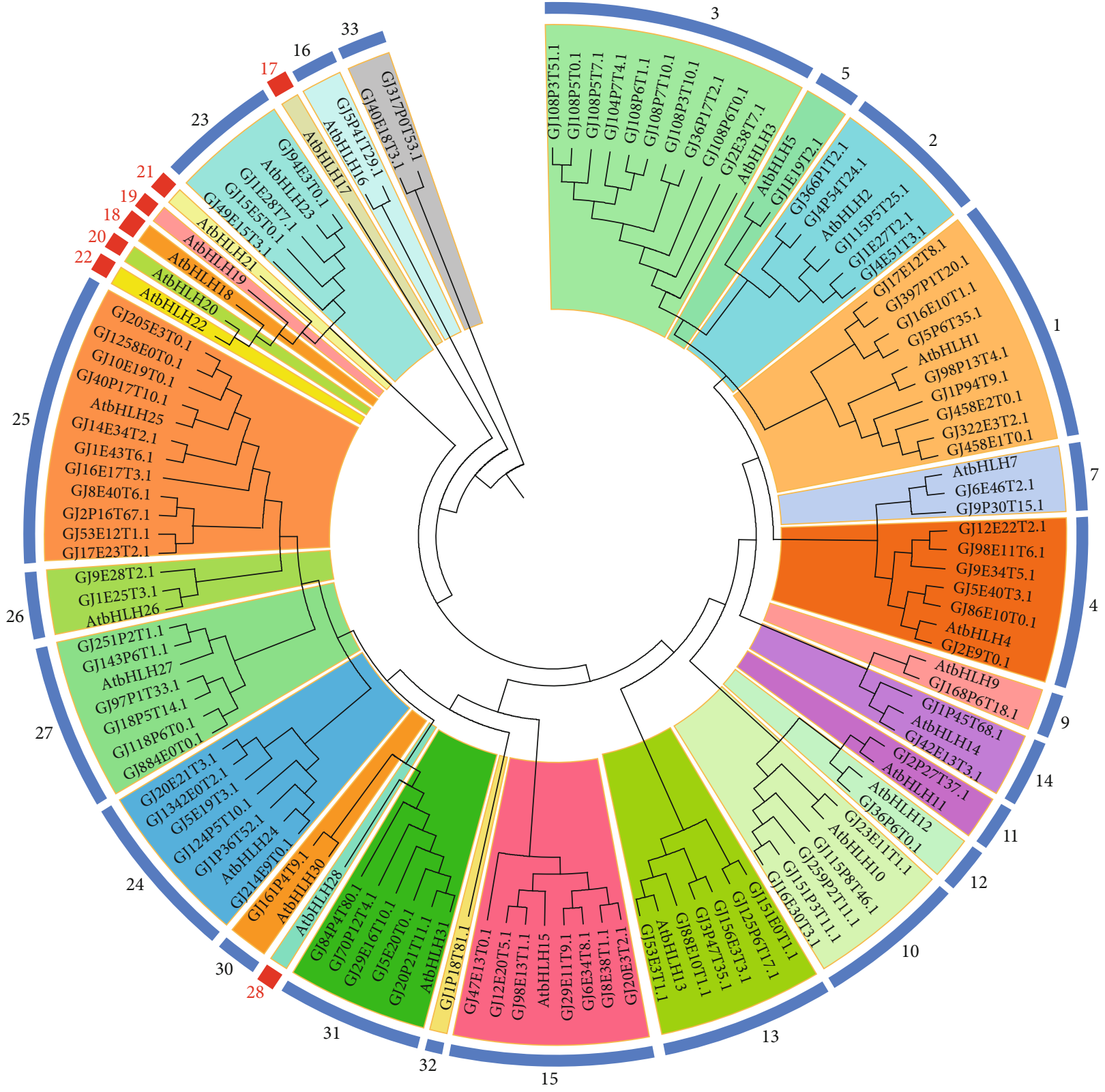

Figure 1: An ML phylogenetic tree of the $b H L H$ transcription factor family in G. jasminoides. Subfamilies 17, 18, 19, 20, 21, 22, and 28 marked in red indicated those commonly existing in A. thaliana, but no GjbHLH genes were found in these subfamilies.

2.5. Promoter Sequence Analysis. The promoter sequences (1500 bp) of key enzyme genes in the crocin biosynthesis pathways were used to predict cis-elements in the New PLACE database (https://www.dna.affrc.go.jp/PLACE/).

\section{Results}

3.1. Gene Prediction and Phylogenetic Analysis. A total of 95 $b H L H s$ were identified that have a complete bHLH domain and named in accordance with the classification principle of Arabidopsis thaliana (Table S3). The GenBank accession numbers of GjbHLH sequences are MN385845 to MN385939 (Table S3). The length of GjbHLH amino acid (aa) sequences varied from 89 aa (GjbHLH25.9) to 966 aa
(GjbHLH23.4). The molecular weights (Mw) of the predicted proteins ranged from 9,904.32 Da (GjbHLH25.9) to $104,750.24 \mathrm{Da}$ (GjbHLH23.4) (Table S4), and the predicted theoretical isoelectric points $(\mathrm{p} I)$ ranged from 4.81 (GjbHLH1.5) to 10.51 (GjbHLH31.4) (Table S4).

The ML phylogenetic tree of the 95 bHLH members in G. jasminoides indicated that 23 distinct subfamilies were identified (Figure 1). The subfamily 25 had the largest number of members (11 GjbHLHs), and subfamilies 5, 9, $11,12,16,30$, and 32 had one GjbHLH gene each. Subfamilies $17,18,19,20,21,22$, and 28 commonly exist in A. thaliana, but no GjbHLH genes were found in these subfamilies. The new subfamilies 32 and 33 were speciesspecific in G. jasminoides. 
In addition, 9 genes, which are distributed in subfamilies 2 and 23, possessed a bHLH-MYC_N (Pfam: PF14215.6) domain, and the other genes have a conserved HLH (Pfam: PF00010.26) domain (Table S3).

3.2. Gene Structure and Conserved Motif Analyses. The structural analyses of the $b H L H$ genes revealed that the number of exons varied from 1 to 12 , and 8 genes were intronless (Figure 2(a)). Subfamily 23 had the most number of exons with an average of 12 . The 8 intronless genes were distributed across 5 subfamilies. Of these genes, 3 belonged to subfamily 31,2 belonged to subfamily 2 , and 3 belonged to subfamilies 1,11 , and 30 (Table S4).

The MEME was used to characterize 22 conserved motifs within bHLH proteins in order to clarify the evolution of bHLH genes (Figure 2(b) and Table S5). Compared with the known HLH and bHLH-MYC_N domains, motif 1 and motif 2 belong to the HLH domain, and motifs 7, 12, and 14 belong to the bHLH-MYC_N domain. GjbHLHs in subfamilies 2 and 3 contained the largest number of motifs (6 types), whereas the GjbHLHs in subfamilies 11 and 16 comprised one motif each (motifs 1 and 2). Additionally, the average number of motifs per $b H L H$ varied across subfamilies and ranged from 1 (subfamilies 11 and 16) to 6 (subfamily 2). Most motifs appeared only once in one bHLH gene, but GjbHLH26.2 protein had two copies of motif 3, and GjbHLH33.1 protein and subfamily 27 have two copies of motif 15 . Motif 1 or 2 was conservatively distributed in all subfamilies. A total of 14 motifs uniquely appear in a specific subfamily: motifs 7 and 16 in subfamily 2; motifs 8,11 , and 13 in subfamily 3; motif 21 in subfamily 4; motifs 5, 14, and 22 in subfamily 23; motifs 18 and 19 in subfamily 24 ; motif 17 in subfamily 27 ; motif 10 in subfamily 31; and motif 19 in subfamily 33 (Figure 3(a)).

The bHLH-MYC_N domain of subfamily 2 was composed of motifs 12 and 7 , and subfamily 23 comprised motifs 12 and 14 (Figures 3(a) and 3(b)). In addition, the ML phylogenetic tree of MYCs from Catharanthus roseus, Nicotiana tabacum, T. chinensis, and A. thaliana and all members of subfamilies 2 and 23 in G. jasminoides indicated that all members of subfamily 2 are clustered with MYC2 (Figure 3(c)). Furthermore, GjbHLH2.3 is clustered with CrMYC2, and GjbHLH2.4 and GjbHLH2.5 are clustered with AtMYC2.3.

3.3. Differential Expression of bHLH Genes in Various Organs. Among the $95 \mathrm{bHLH}$ genes, the expression of 13 $b H L H$ genes distributed across 8 subfamilies was undetected, with FPKM values of less than 1 (Figure 4, Table S6). The expression of the other $72 \mathrm{bHLH}$ genes comprising 19 subfamilies was detected, with FPKM values higher than 1 in at least one of the six organs tested. In addition, 44 GjbHLH genes showed a significantly high expression ( $\log 2 \mid$ fold change $\mid>1$ ) in at least one of the organs (root, stem, leaf, and young fruit), and 13 genes exhibited a significantly high expression in all four organs. The expression pattern of $\mathrm{Gj} b H \mathrm{HH}$ genes indicated that more GjbHLH genes showed a relatively low expression in mature G. jasminoides fruits. A total of 13 genes were specifically expressed in six organs. In particular, 7 genes (GjbHLH1.3, GjbHLH2.1, GjbHLH3.1, GjbHLH3.4, GjbHLH3.10, GjbHLH10.4, and GjbHLH31.2) were specifically expressed in the root, 2 genes (GjbHLH15.2 and GjbHLH25.1) were specifically expressed in the stem, and only 1 gene was expressed specifically in the leaf (GjbHLH7.2), young fruit (GjbHLH25.9), green fruit (GjbHLH15.4), and red fruit (GjJbHLH1.8).

Crocins have been indicated to be highly accumulated in mature G. jasminoides fruits [37]. The expression of the key enzyme genes, including GjCCD4a, GjALDH2C3, GjUGT94E13, and GjUGT74F8, in the crocin biosynthesis pathway was higher in mature fruits than in other organs. However, the expression of 9 genes (GjbHLH1.7, GjbHLH1.9, GjbHLH2.2, GjbHLH2.3, GjbHLH2.4, GjbHLH2.5, GjbHLH3.10, GjbHLH7.2, and GjbHLH27.3) significantly decreased in mature fruits compared with those in the four other organs; the FPKM values were higher than 50 in the four other organs and at least 2 -fold higher than those in red fruit. Among them, 4 genes (GjbHLH2.2, GjbHLH2.3, GjbHLH2.4, and GjbHLH2.5) belonged to the MYC2 family. The qRTPCR analysis of the 9 candidate genes indicated that the expression of 7 genes in red fruits was significantly low, which was consistent with the RNA-Seq results (Figure 5, Table S7).

3.4. cis-Acting Elements of Promoter of Crocin Biosynthetic Genes. The analysis of the promoter sequences of 6 key enzyme genes (GjBCH, GjLCYB, GjALDH2C3, GjUGT94E13, GjUGT74F8, and CjCCD4a) in the crocin biosynthesis pathway and candidate GjbHLH genes showed that all the promoters contained E-box sites, which are the regulatory elements of bHLH TFs (Tables S8 and S9). The promoters of 6 key enzyme genes have 7, 3, 4, 5, 9, and 4 E-box binding sites. In addition, 10 classic MYC2-binding cisacting elements, including 4 AACGTG (T/G-box), 1 CACG TG (G-box), and 5 CACATG (MYCATRD22), were found in the promoters of crocin biosynthetic genes. Moreover, 5 MYCATRD22 elements were found in the promoters of GjLCYB, GjBCH, GjALDH2C3, and GjUGT74F8, 4 T/G-box elements were detected in the promoters of GjALDH2C3, GjUGT94E13, and GjCCD4a, and 1 G-box element was observed in the promoter of GjUGT74E8. Furthermore, 6 candidates of GjbHLH genes (GjbHLH2.3, GjbHLH2.4, GjbHLH2.5, GjbHLH3.10, GjbHLH7.2, and GjbHLH27.3) contained at least one of the elements of T/G-box and MYCATRD22.

\section{Discussion}

bHLH TFs play an important role in plant stress resistance [38], signal transduction [39], secondary metabolism regulation [40], and growth and development [41]. They have been identified and analyzed in various plants. According to the sequence homology and phylogenetic relationships, 167, $183,177,169$, and $127 \mathrm{bHLH}$ genes have been identified in A. thaliana [30], P. trichocarpa [30], O. sativa [30], P. ginseng [20], and S. miltiorrhiza [19], respectively. Here, we first identified 95 GjbHLH TF genes in G. jasminoides and classified them into 23 subfamilies. The gene number of bHLH 


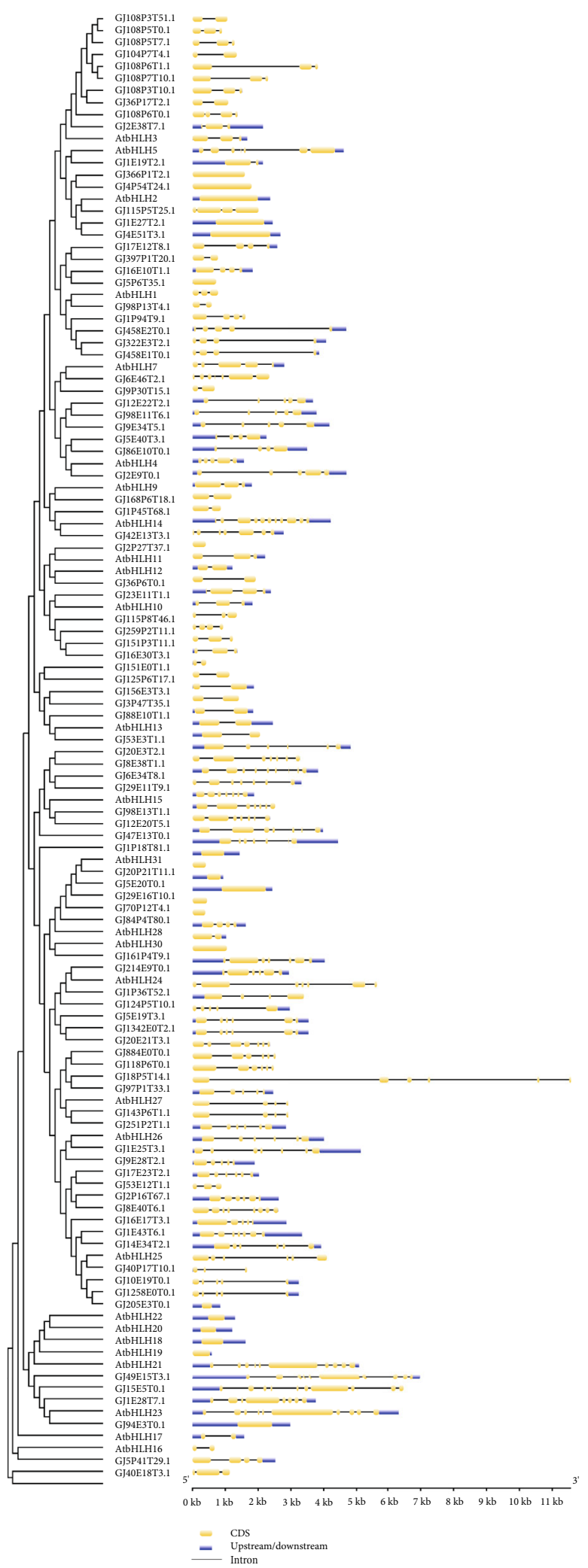

(a)

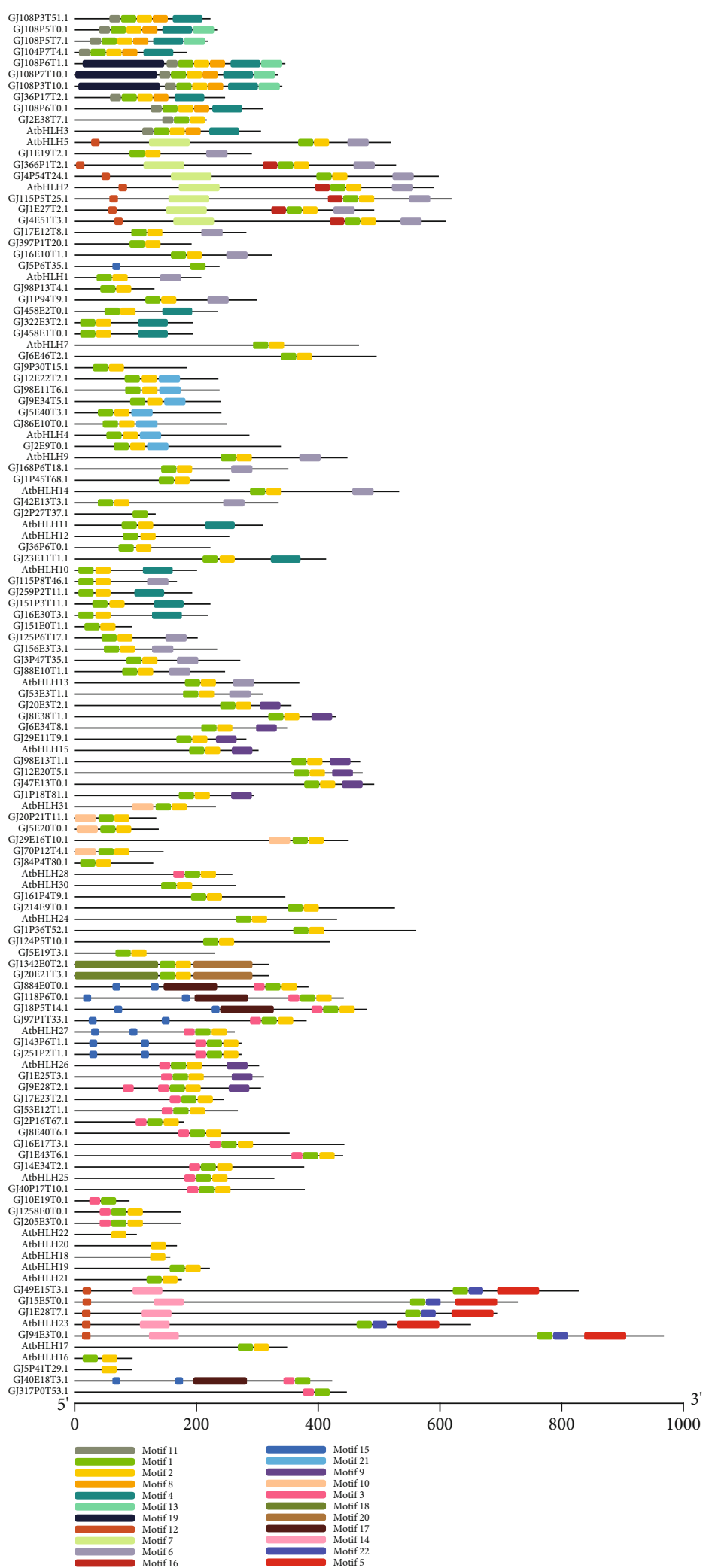

(b)

Figure 2: The structural features and conserved motif analysis of GjbHLH genes. (a) The structural features of each $b H L H$ gene in $G$. jasminoides. Exons and UTRs are represented by yellow and blue round-cornered rectangles, respectively. Introns are shown by black connecting lines. (b) The distribution of conserved motifs in each bHLH gene of G. jasminoides. The relative positions of each conserved motif within the bHLH protein are shown in color. 


\begin{tabular}{|c|c|c|c|c|c|c|c|c|c|c|c|c|c|c|c|c|c|c|c|c|c|c|c|}
\hline & \multicolumn{23}{|c|}{ Subfamily } \\
\hline & 1 & 2 & 3 & 4 & 5 & 7 & 9 & 10 & 11 & 12 & 13 & 14 & 15 & 16 & 23 & 24 & 25 & 26 & 27 & 30 & 31 & 32 & 33 \\
\hline MOTIF 1 & - & - & - & - & - & - & - & - & - & - & - & - & - & & - & - & - & - & - & - & - & - & - \\
\hline MOTIF 2 & - & - & - & - & - & - & - & - & & - & - & - & - & - & & - & - & - & - & - & - & - & \\
\hline MOTIF 3 & & & & & & & & & & & & & & & & & - & - & - & & & & - \\
\hline MOTIF 4 & - & & - & & & & & - & & & & & & & & & & & & & & & \\
\hline MOTIF 5 & & & & & & & & & & & & & & & - & & & & & & & & \\
\hline MOTIF 6 & - & - & & & - & & - & - & & & - & & & & & & & & & & & & \\
\hline MOTIF 7 & & & - & & & & & & & & & & & & & & & & & & & & \\
\hline MOTIF 8 & & & - & & & & & & & & & & & & & & & & & & & & \\
\hline MOTIF 9 & & & & & & & & & & & & & - & & & & & - & & & & - & \\
\hline MOTIF 10 & & & & & & & & & & & & & & & & & & & & & - & & \\
\hline MOTIF 11 & & & & & & & & & & & & & & & & & & & & & & & \\
\hline MOTIF 12 & & - & & & & & & & & & & & & & - & & & & & & & & \\
\hline MOTIF 13 & & & - & & & & & & & & & & & & & & & & & & & & \\
\hline MOTIF 14 & & & & & & & & & & & & & & & - & & & & & & & & \\
\hline MOTIF 15 & & & & & & & & & & & & & & & & & & & & & & & \\
\hline MOTIF 16 & & & & & & & & & & & & & & & & & & & & & & & \\
\hline MOTIF 17 & & & & & & & & & & & & & & & & & & & & & & & \\
\hline MOTIF 18 & & & & & & & & & & & & & & & & - & & & & & & & \\
\hline MOTIF 19 & & & & & & & & & & & & & & & & & & & & & & & \\
\hline MOTIF 20 & & & & & & & & & & & & & & & & - & & & & & & & \\
\hline MOTIF 21 & & & & - & & & & & & & & & & & & & & & & & & & \\
\hline MOTIF 22 & & & & & & & & & & & & & & & & & & & & & & & \\
\hline
\end{tabular}

(a)

Motif 12

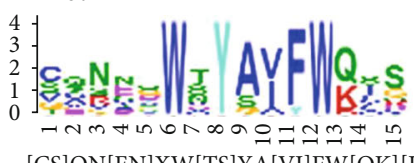

Motif 7

bHLH-MYC_N domain of subfamily 2

(b)

Figure 3: Continued. 


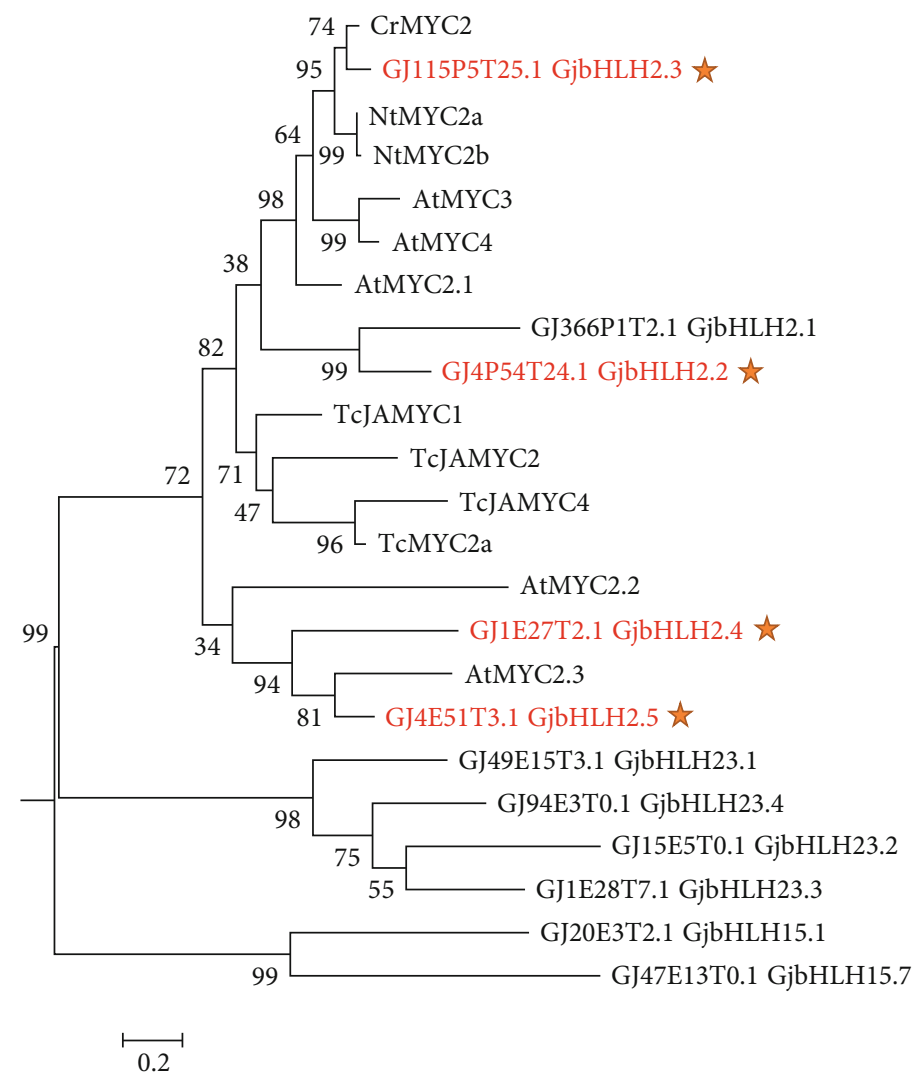

(c)

Figure 3: The distribution analysis of conserved motifs of GjbHLH genes in each subfamily and the phylogenetic tree and motif analysis of subfamily 2. (a) Distribution of 22 motifs in each subfamily. (b) The bHLH-MYC_N domain of subfamily 2 consists of motifs 12 and 7. (c) An ML phylogenetic tree was constructed with the 12 MYC2 genes from C. roseus, N. tabacum, T. chinensis, and A. thaliana and 9 MYCs from G. jasminoides. GjbHLH15.1 and GjbHLH15.7 branches were chosen as an outgroup. The MYC2s were downloaded with the sequence numbers: CrMYC2 (AF283507); NtMYC2a (HM466974) and NtMYC2b (HM466975); TcJAMYC2 (JX519289), TcJAMYC4 (JX519290), and TcJAMYC1 (FJ608574); TcMYC2a (MG494378); and AtMYC3 (AT5G46760), AtMYC4 (AT4G17880), AtMYC2.1 (AT1G32640), AtMYC2.2 (AT1G63650), and AtMYC2.3 (AT1G01260).

genes is similar with that in the $V$. vinifera genome (94 genes) [21]. The variable numbers of bHLH genes in these species are probably caused by whole genome duplication or tandem repeat during plant evolution. Both V. vinifera and G. jasminoides did not experience a whole genome duplication event after the ancient gamma triplication event of eudicots [42, 43]; however, the genomes of $A$. thaliana, P. trichocarpa, $O$. sativa, $P$. ginseng, and $S$. miltiorrhiza underwent at least one whole genome duplication event after a shared gamma event [44]. Thence, the bHLH genes in these species showed significant expansion, compared with $V$. vinifera and $G$. jasminoides. In addition, compared with the bHLH family of Arabidopsis, the members of subfamilies 17, 18, 19, 20, 21, 22 , and 28 of bHLH TFs are lost in the G. jasminoides genome, and two new subfamilies 32 and 33 were identified in G. jasminoides. Gene families in plants might primarily evolve through tandem duplication [45]. In the G. jasminoides genome, we identified one tandem gene duplication, covering $7 \mathrm{bHLH}$ genes in scaffold 108. All the clustered $b H L H$ genes belonged to subfamily 3, suggesting the expansion of subfamily 3 members in G. jasminoides. The phylogenetic analysis of bHLH3 between $G$. jasminoides and previous reports of bHLH3 family members from other plants suggested that the expansion of subfamily 3 was species-specific after the speciation of $G$. jasminoides (Figure S2).

bHLH TFs directly or indirectly regulate the biosynthesis of active compounds in medicinal plants. For example, SmbHLH10 overexpression in S. miltiorrhiza enhanced the accumulation of tanshinones [46]. In Medicago truncatula, two bHLH TFs, TSARL1 and TSARL2, positively increase the expression of the triterpene biosynthetic genes, resulting in triterpene saponin accumulation [47]. In G. jasminoides, the expression profile of 9 candidate $b H L H$ TFs in matured fruits significantly decreased and negatively correlated with the expression of GjCCD4a, GjALDH2C3, UGT74F8, and UGT94E13. Among them, 4 genes (GjbHLH2.2, GjbHLH2.3, GjbHLH2.4, and GjbHLH2.5) had bHLH-MYC_N protein domains. Promoter sequence analysis indicated that the 6 key enzyme genes in crocin biosynthesis pathways had a large number of bHLH TF binding sites, suggesting the potential regulation of $b H L H s$ in G. jasminoides. The promoters of 7 candidate $\mathrm{Gj} b H L H$ genes contained at least one of the motifs of T/G-box, G-box, and MYCATRD22 that 


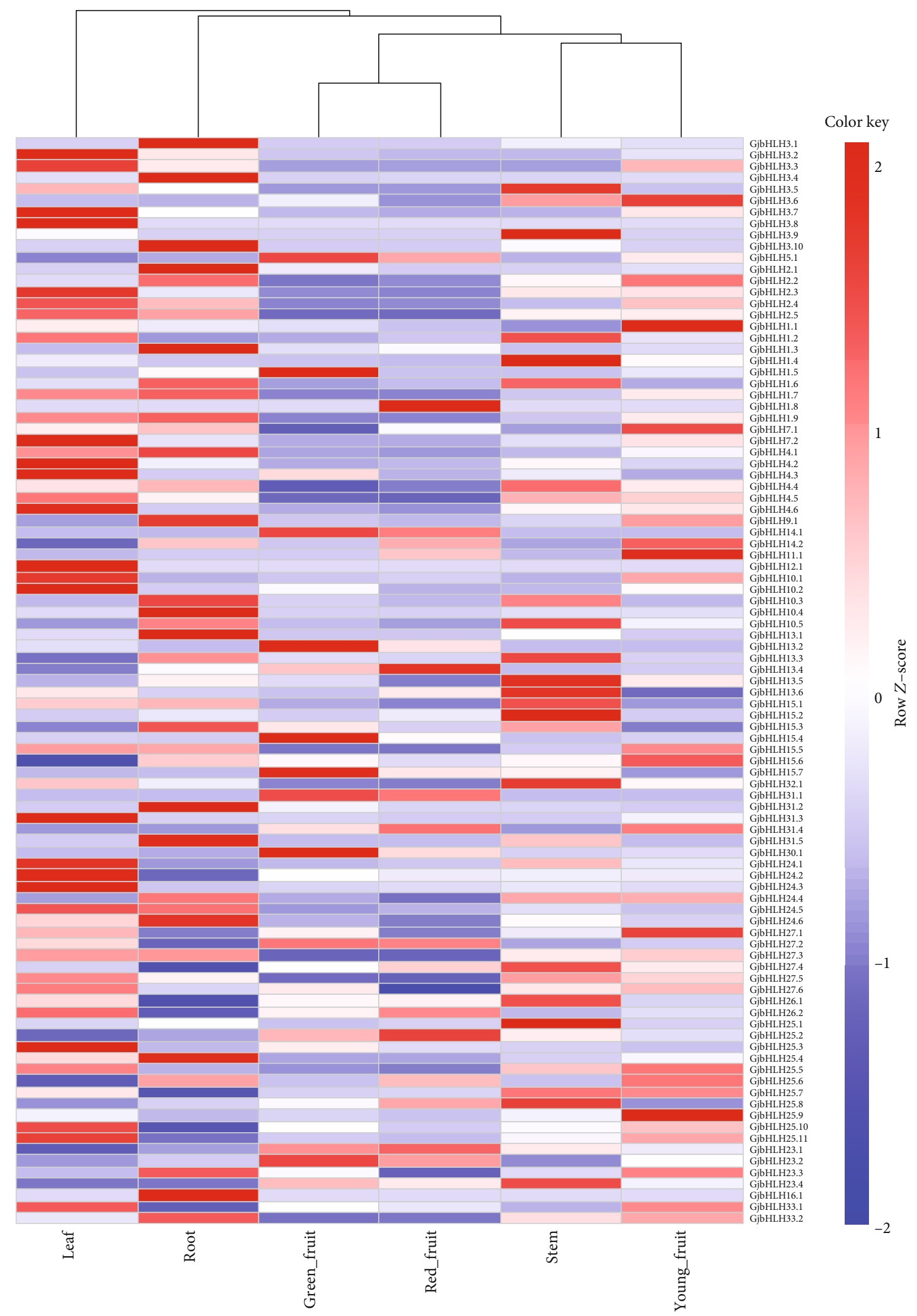

FIGURE 4: Heatmaps representing the expression profiles of G. jasminoides bHLH genes in the root, stem, leaf, young fruit, green fruit, and red fruit. The FPKM value was normalized using $Z$-score (row). 
GjbHLH1.7

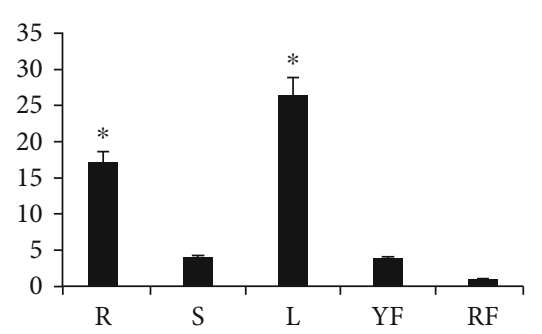

GjbHLH2.3

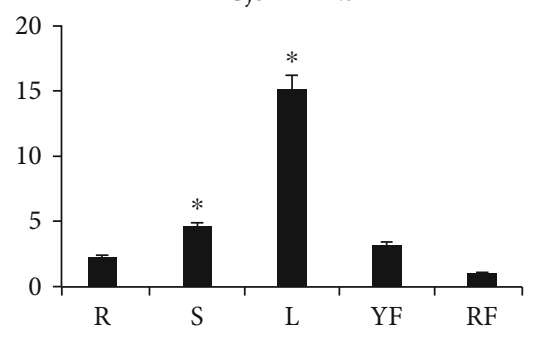

GjbHLH3.10

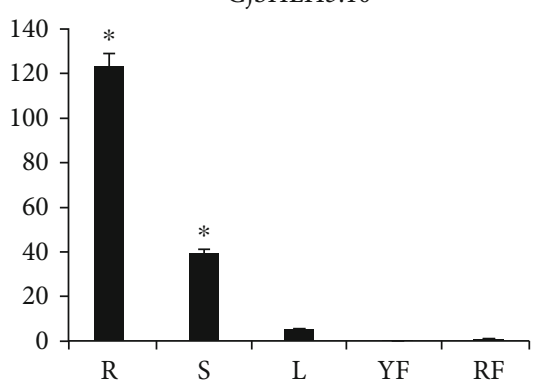

GjbHLH1.9

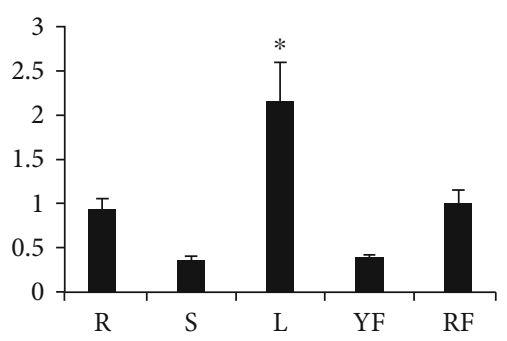

GjbHLH2.4

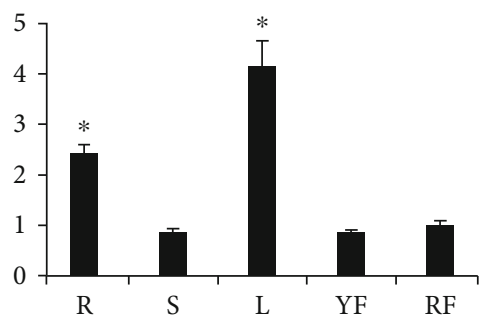

GjbHLH7.2

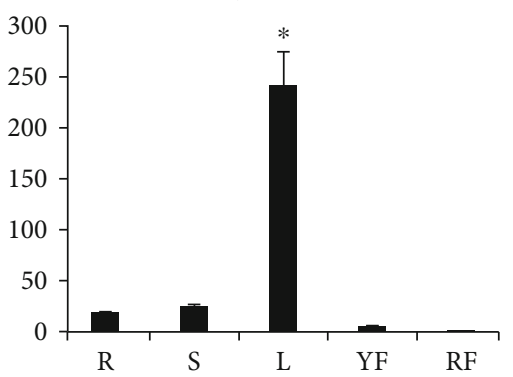

GjbHLH2.2

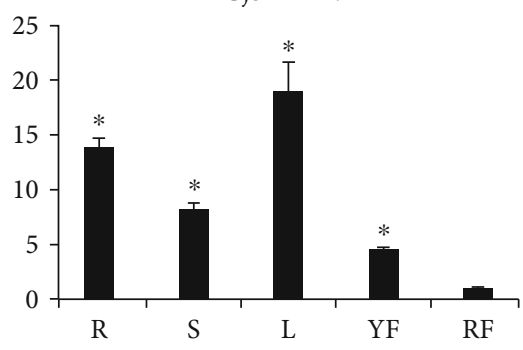

GjbHLH2.5

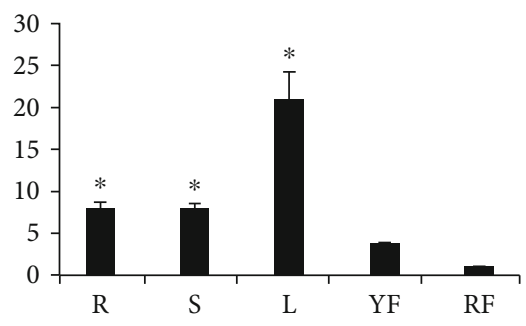

GjbHLH27.3

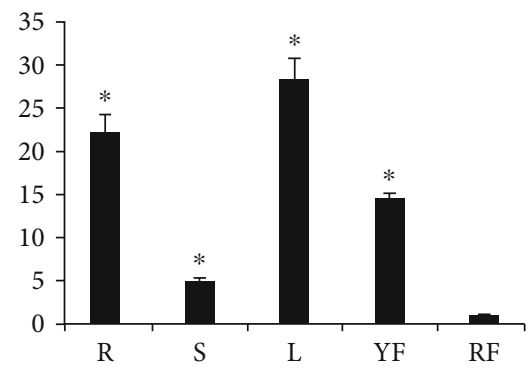

FiguRE 5: qRT-PCR of nine candidate $b H L H$ genes related to the regulation of crocin biosynthesis. The characters on the $x$-axis indicate the root $(\mathrm{R})$, stem $(\mathrm{S})$, leaf $(\mathrm{L})$, young fruit $(\mathrm{YF})$, and red fruit $(\mathrm{RF})$. The $y$-axis represents the relative level of gene expression. The actin gene was used as an internal reference. One-way ANOVA was performed with GraphPad Prism. Asterisks $(*)$ represent significant differences with the red fruit. ${ }^{*} P<0.05$ was considered a significant level.

could be bound by MYC2. The phylogenetic analysis of MYCs revealed that GjbHLH2.3 is clustered with CrMYC2, which can regulate the biosynthesis of medicinally valuable terpenoid indole alkaloids in C. roseus [48]. Furthermore, the qPCR analysis indicated that the expression of most candidate genes in mature fruits was significantly low, which was consistent with the RNA-Seq results. These results implied that $b H L H$ TFs might negatively regulate the biosynthesis of crocins in G. jasminoides. Candidate bHLH TFs should be functionally verified to clarify the molecular mechanism of the regulation of crocin biosynthesis.

\section{Conclusions}

In conclusion, 95 bHLH TF genes were identified and phylogenetic analyzed in the genome of G. jasminoides. These GjbHLHs could be classified into 23 subfamilies supported by phylogeny, additional protein motifs, and intron/exon structures. Gene-specific expression patterns, crocin biosynthesis pathways, and elucidation of the complete pathway of the crocin biosynthesis in mature G. jasminoides fruits revealed that $9 \mathrm{bHLH}$ TF genes identified in G. jasminoides were potentially involved in the regulation of crocin biosyn- thesis. The candidate $b H L H$ TF genes related to crocin biosynthesis should be further functionally identified via a series of in vivo and in vitro experimental procedures.

\section{Data Availability}

The bHLH transcription factor sequences of G. jasminoides have been uploaded into NCBI database, and the GenBank accession numbers of GjbHLH sequences are from MN385845 to MN385939.

\section{Conflicts of Interest}

The authors declare that there are no conflicts of interest regarding the publication of this paper.

\section{Acknowledgments}

The authors thank Prof. Kaizhi Hu (Chongqing Institute of Medicinal Plant Cultivation) for providing the Gardenia jasminoides material (ZZ1-9) used in this work. We also thank Prof. Jingyuan Song for comments on the manuscript. This research was supported by the National Natural Science 
Foundation of China (Grant no. 81973424), the CAMS Innovation Fund for Medical Sciences (CIFMS) (2016-I2M3-016), the Construction Program of Biology First-Class Discipline in Guizhou (GNYL [2017]009), and the Guizhou Province Medicinal Plant Breeding and Planting Talent Base (Guizhou Ren Ling FA [2013] No. 15).

\section{Supplementary Materials}

Supplementary 1. Figure S1: the potential crocin biosynthetic pathway in G. jasminoides.

Supplementary 2. Figure S2: an ML phylogenetic tree was constructed with GjbHLH3 subfamily and bHLH3 family members reported from other plants. GjbHLH15.1 and GjbHLH15.7 branches were chosen as the outgroup. bHLH3 family members were downloaded with the following sequence numbers: AT2G22770, AT2G22750, AT2G22760, AT4G37850, OS03G51580, OS03G12760, OS03G46860, OS12G43620, OS03G46790, and OS10G01530.

Supplementary 3. Table S1: the accession numbers of AtbHLH genes. Table S2: primer sequences of candidate GjbHLH genes. Table S3: identification of GjbHLH genes through BLAST and HMMER analysis of the $b H L H$ domain against the G. jasminoides genome. Table S4: analysis of the structural features and conserved motifs of $b H L H$ genes in G. jasminoides. Table S5: conserved motifs predicted by MEME. Table S6: the FPKM values of $95 \mathrm{GjbHLH}$ genes in different organs. Asterisks $(*)$ represent the coexpression of four genes (GjALDH2C3, GjUGT94E13, GjUGT74F8, and CjCCD4a) in crocin biosynthesis. Table S7: the qRT-PCR analysis for candidate GjbHLH genes. Table S8: the cis-acting elements of the promoter sequences of 6 key enzyme genes (GjBCH, GjLCYB, GjALDH2C3, GjUGT94E13, GjUGT74F8, and $C j C C D 4 a$ ) in the crocin biosynthesis pathway. Table S9: the cis-acting elements of the promoter sequences of candidate GjbHLH genes.

\section{References}

[1] Y. Ni, L. Li, W. Zhang et al., "Discovery and LC-MS characterization of new crocins in Gardeniae Fructus and their neuroprotective potential," Journal of Agricultural and Food Chemistry, vol. 65, no. 14, pp. 2936-2946, 2017.

[2] T. Farkhondeh, S. Samarghandian, H. Shaterzadeh Yazdi, and F. Samini, "The protective effects of crocin in the management of neurodegenerative diseases: a review," American Journal of Neurodegenerative Disease, vol. 7, no. 1, pp. 1-10, 2018.

[3] M. N. Shafei, A. Faramarzi, A. Khajavi Rad, and A. Anaeigoudari, "Crocin prevents acute angiotensin IIinduced hypertension in anesthetized rats," Avicenna journal of phytomedicine, vol. 7, no. 4, pp. 345-352, 2017.

[4] A. Colapietro, A. Mancini, A. M. D'Alessandro, and C. Festuccia, "Crocetin and crocin from saffron in cancer chemotherapy and chemoprevention," Anti-Cancer Agents in Medicinal Chemistry, vol. 19, no. 1, pp. 38-47, 2019.

[5] A. Amin, A. A. Hamza, S. Daoud et al., "Saffron-based crocin prevents early lesions of liver cancer: in vivo, in vitro and network analyses," Recent Patents on Anti-Cancer Drug Discovery, vol. 11, no. 1, pp. 121-133, 2016.
[6] H. Liu, Y. F. Chen, F. Li, and H. Y. Zhang, "Fructus Gardenia (Gardenia jasminoides J. Ellis) phytochemistry, pharmacology of cardiovascular, and safety with the perspective of new drugs development," Journal of Asian Natural Products Research, vol. 15, no. 1, pp. 94-110, 2013.

[7] M. Gu, L. Luo, and K. Fang, "Crocin inhibits obesity via AMPK-dependent inhibition of adipocyte differentiation and promotion of lipolysis," Bioscience Trends, vol. 12, no. 6, pp. 587-594, 2018.

[8] E. Ouyang, C. Zhang, and X. Li, "Simultaneous determination of geniposide, chlorogenic acid, crocin 1, and rutin in crude and processed Fructus Gardeniae extracts by high performance liquid chromatography," Pharmacognosy Magazine, vol. 7, no. 28, pp. 267-270, 2011.

[9] Z. Mei, M. A. Khan, L. Yang, M. Yang, and J. Fu, "Genetic characterization and authentication of Gardenia jasminoides in different regions of China by using improved RAPD analysis," Indian Journal of Experimental Biology, vol. 53, no. 3, pp. 164-169, 2015.

[10] T. Xin, Y. Zhang, X. Pu, R. Gao, Z. Xu, and J. Song, "Trends in herbgenomics," Life Sciences, vol. 62, no. 3, pp. 288-308, 2019.

[11] L. Liu, M. J. White, and T. H. MacRae, "Transcription factors and their genes in higher plants functional domains, evolution and regulation," European Journal of Biochemistry, vol. 262, no. 2, pp. 247-257, 1999.

[12] Y. Zhang, A. Ji, Z. Xu, H. Luo, and J. Song, "The AP2/ERF transcription factor SmERF128 positively regulates diterpenoid biosynthesis in Salvia miltiorrhiza," Plant Molecular Biology, vol. 100, no. 1-2, pp. 83-93, 2019.

[13] Z. Xu and J. Song, "The 2-oxoglutarate-dependent dioxygenase superfamily participates in tanshinone production in Salvia miltiorrhiza," Journal of Experimental Botany, vol. 68, no. 9, pp. 2299-2308, 2017.

[14] A. J. Ji, H. M. Luo, Z. C. Xu et al., "Genome-wide identification of the AP2/ERF gene family involved in active constituent biosynthesis in Salvia miltiorrhiza," The Plant Genome, vol. 9, no. 2, 2016.

[15] M. A. Heim, M. Jakoby, M. Werber, C. Martin, B. Weisshaar, and P. C. Bailey, "The basic helix-loop-helix transcription factor family in plants: a genome-wide study of protein structure and functional diversity," Molecular Biology and Evolution, vol. 20, no. 5, pp. 735-747, 2003.

[16] X. Li, X. Duan, H. Jiang et al., "Genome-wide analysis of basic/helix-loop-helix transcription factor family in rice and Arabidopsis," Plant Physiology, vol. 141, no. 4, pp. 1167-1184, 2006.

[17] J. Wang, Z. Hu, T. Zhao et al., "Genome-wide analysis of bHLH transcription factor and involvement in the infection by yellow leaf curl virus in tomato (Solanum lycopersicum)," BMC Genomics, vol. 16, no. 1, p. 39, 2015.

[18] X. M. Song, Z. N. Huang, W. K. Duan et al., "Genome-wide analysis of the bHLH transcription factor family in Chinese cabbage (Brassica rapa ssp. pekinensis)," Molecular Genetics and Genomics, vol. 289, no. 1, pp. 77-91, 2014.

[19] X. Zhang, H. Luo, Z. Xu et al., "Genome-wide characterisation and analysis of bHLH transcription factors related to tanshinone biosynthesis in Salvia miltiorrhiza," Scientific Reports, vol. 5, no. 1, article 11244, 2015.

[20] Y. Chu, S. Xiao, H. Su et al., "Genome-wide characterization and analysis of bHLH transcription factors in Panax ginseng," Acta Pharmaceutica Sinica B, vol. 8, no. 4, pp. 666-677, 2018. 
[21] P. Wang, L. Su, H. Gao et al., "Genome-wide characterization of bHLH genes in grape and analysis of their potential relevance to abiotic stress tolerance and secondary metabolite biosynthesis," Frontiers in Plant Science, vol. 9, p. 64, 2018.

[22] K. Mao, Q. Dong, C. Li, C. Liu, and F. Ma, "Genome wide identification and characterization of apple bHLH transcription factors and expression analysis in response to drought and salt stress," Frontiers in Plant Science, vol. 8, p. 480, 2017.

[23] C. Gao, J. Sun, C. Wang et al., "Genome-wide analysis of basic/helix-loop-helix gene family in peanut and assessment of its roles in pod development," PLoS One, vol. 12, no. 7, article e0181843, 2017.

[24] M. Zhang, X. Jin, Y. Chen et al., "TcMYC2a, a basic helix-loophelix transcription factor, Transduces JA-Signals and Regulates Taxol Biosynthesis in Taxus chinensis," Frontiers in Plant Science, vol. 9, p. 863, 2018.

[25] B. Dombrecht, G. P. Xue, S. J. Sprague et al., "MYC2 differentially modulates diverse jasmonate-dependent functions in Arabidopsis," The Plant Cell, vol. 19, no. 7, pp. 2225-2245, 2007.

[26] S. K. Lenka, N. E. Nims, K. Vongpaseuth, R. A. Boshar, S. C. Roberts, and E. L. Walker, "Jasmonate-responsive expression of paclitaxel biosynthesis genes in Taxus cuspidata cultured cells is negatively regulated by the bHLH transcription factors TcJAMYC1, TcJAMYC2, and TcJAMYC4," Frontiers in Plant Science, vol. 6, p. 115, 2015.

[27] O. Ahrazem, J. Argandona, A. Fiore et al., "Multi-species transcriptome analyses for the regulation of crocins biosynthesis in crocus," BMC Genomics, vol. 20, no. 1, p. 320, 2019.

[28] X. Zhang, F. Ge, B. Deng et al., "Molecular cloning and characterization of PnbHLH1 transcription factor in Panax notoginseng," Molecules, vol. 22, no. 8, article 1268, 2017.

[29] Y. Li, P. Xu, G. Chen, J. Wu, Z. Liu, and H. Lian, "FvbHLH9, functions as a positive regulator of anthocyanin biosynthesis, by forming HY5-bHLH9 transcription complex in strawberry fruits," Plant \& Cell Physiology, 2020.

[30] L. Carretero-Paulet, A. Galstyan, I. Roig-Villanova, J. F. Martinez-Garcia, J. R. Bilbao-Castro, and D. L. Robertson, "Genome-wide classification and evolutionary analysis of the bHLH family of transcription factors in Arabidopsis, poplar, rice, moss, and algae," Plant Physiology, vol. 153, no. 3, pp. 1398-1412, 2010.

[31] R. C. Edgar, "MUSCLE: a multiple sequence alignment method with reduced time and space complexity," BMC Bioinformatics, vol. 5, no. 1, p. 113, 2004.

[32] K. Tamura, G. Stecher, D. Peterson, A. Filipski, and S. Kumar, "MEGA6: molecular evolutionary genetics analysis version 6.0," Molecular Biology and Evolution, vol. 30, no. 12, pp. 2725-2729, 2013.

[33] T. L. Bailey, M. Boden, F. A. Buske et al., "MEME Suite: tools for motif discovery and searching," Nucleic Acids Research, vol. 37, pp. W202-W208, 2009.

[34] D. Kim, J. M. Paggi, C. Park, C. Bennett, and S. L. Salzberg, "Graph-based genome alignment and genotyping with HISAT2 and HISAT-genotype," Nature Biotechnology, vol. 37, no. 8, pp. 907-915, 2019.

[35] C. Trapnell, D. G. Hendrickson, M. Sauvageau, L. Goff, J. L. Rinn, and L. Pachter, "Differential analysis of gene regulation at transcript resolution with RNA- seq," Nature Biotechnology, vol. 31, no. 1, pp. 46-53, 2013.
[36] Z. Xu, A. Ji, J. Song, and S. Chen, "Genome-wide analysis of auxin response factor gene family members in medicinal model plant Salvia miltiorrhiza," Biology open, vol. 5, no. 6, pp. 848-857, 2016.

[37] A. Ji, J. Jia, Z. Xu et al., "Transcriptome-guided mining of genes involved in crocin biosynthesis," Frontiers in Plant Science, vol. 8, p. 518, 2017.

[38] X. Sun, Y. Wang, and N. Sui, "Transcriptional regulation of bHLH during plant response to stress," Biochemical and Biophysical Research Communications, vol. 503, no. 2, pp. 397401, 2018.

[39] R. Lu, J. Zhang, D. Liu, Y. L. Wei, Y. Wang, and X. B. Li, "Characterization of bHLH/HLH genes that are involved in brassinosteroid (BR) signaling in fiber development of cotton (Gossypium hirsutum)," BMC Plant Biology, vol. 18, no. 1, p. 304, 2018.

[40] W. R. Chezem and N. K. Clay, "Regulation of plant secondary metabolism and associated specialized cell development by MYBs and bHLHs," Phytochemistry, vol. 131, pp. 26-43, 2016.

[41] C. X. Wang, C. Y. Qi, J. H. Luo, L. Liu, Y. He, and L. Q. Chen, "Characterization of LRL5 as a key regulator of root hair growth in maize," The Plant Journal, vol. 98, no. 1, pp. 7182, 2019.

[42] The French-Italian Public Consortium for Grapevine Genome Characterization, "The grapevine genome sequence suggests ancestral hexaploidization in major angiosperm phyla," Nature, vol. 449, no. 7161, pp. 463-467, 2007.

[43] F. Denoeud, L. Carretero-Paulet, A. Dereeper et al., "The coffee genome provides insight into the convergent evolution of caffeine biosynthesis," Science, vol. 345, no. 6201, pp. 1181-1184, 2014.

[44] Y. Van de Peer, E. Mizrachi, and K. Marchal, "The evolutionary significance of polyploidy," Nature Reviews Genetics, vol. 18, no. 7, pp. 411-424, 2017.

[45] S. B. Cannon, A. Mitra, A. Baumgarten, N. D. Young, and G. May, "The roles of segmental and tandem gene duplication in the evolution of large gene families in Arabidopsis thaliana," BMC Plant Biology, vol. 4, no. 1, p. 10, 2004.

[46] B. Xing, D. Yang, H. Yu et al., "Overexpression of SmbHLH10 enhances tanshinones biosynthesis in Salvia miltiorrhiza hairy roots," Plant Science, vol. 276, pp. 229-238, 2018.

[47] J. Mertens, J. Pollier, R. Vanden Bossche, I. Lopez-Vidriero, J. M. Franco-Zorrilla, and A. Goossens, "The bHLH transcription factors TSAR1 and TSAR2 regulate triterpene saponin biosynthesis in Medicago truncatula," Plant Physiology, vol. 170, no. 1, pp. 194-210, 2016.

[48] H. Zhang, S. Hedhili, G. Montiel et al., "The basic helix-loophelix transcription factor CrMYC2 controls the jasmonateresponsive expression of the ORCA genes that regulate alkaloid biosynthesis in Catharanthus roseus," The Plant Journal, vol. 67 , no. 1, pp. 61-71, 2011. 